\title{
Assessing social competence in visually impaired people and proposing an interventional program in visually impaired children
}

\author{
Giulia Cappagli $^{1^{*}}$, Sara Finocchietti ${ }^{1 *}$, Gabriel Baud-Bovy ${ }^{2,3}$, Leonardo Badino ${ }^{4}$, Alessandro \\ D'Ausilio $^{4,5}$, Elena Cocchi ${ }^{6}$, Monica Gori ${ }^{1}$
}

\begin{abstract}
Visually impaired children and adults have difficulties in engaging in positive social interactions. This study assesses social competence in sighted and visually impaired people and to propose a novel interventional strategy in visually impaired children.

We designed a task that assesses the ability to initiate and sustain an interaction with the experimenter while performing free hand movements using a sonorous feedback on the experimenter's wrist. Both participant and experimenter kinematic data were recorded with a motion capture system. The level of social interaction between participant and experimenter has been computed through objective measurements based on Granger causality analysis applied to the participant arm kinematics.

The interventional program followed by the visually impaired children lasted 12 weeks and consisted in a series of spatial and social games performed with the use of a sonorous bracelet which provides an auditory feedback of body actions in space.

Visually impaired individuals present a poorer communication flow with the experimenter than sighted people, which indicates a less efficient social interaction. The amount of communication between the two agents resulted in a significant improvement after the interventional program. Thus, a specific intervention, based on the substitution of visual with auditory feedback of body actions, can enhance social inclusion for the blind population.
\end{abstract}

Index Terms - social interaction, blindness, Granger causality

This research has been partially supported by the EU Strep project ABBI (FP7-ICT 611452) and EU Irses project CODEFROR (FP7-PIRSES-2013612555).

*Giulia Cappagli and Sara Finocchietti equally contributed to the research article.

1 Unit for Visually Impaired People (U-VIP), Istituto Italiano di Tecnologia, Genova, Italy.

2 Robotics, Brain and Cognitive Science (RBCS), Istituto Italiano di Tecnologia, Genova, Italy.

3 Vita-Salute San Raffaele University \& Unit of Experimental Psychology, Division of Neuroscience, San Raffaele Scientific Institute, Milan, Italy

4 Center for Translational Neurophysiology of Speech and Communication (CTNSC), Istituto Italiano di Tecnologia, Ferrara, Italy.

5 Università di Ferrara, Dip. Scienze Biomediche e Chirurgico Specialistiche, Sez. Fisiologia Umana, Ferrara, Italy. Italy

6 Istituto David Chiossone per Ciechi ed ipovedenti ONLUS, Genova,

Corresponding author: Monica.gori@iit.it.

\section{INTRODUCTION}

OCIAL cognition is the product of the behavioral and cognitive processes dedicated to build our social world. In humans as well as in other species, many social cues take the form of body language and therefore require vision in order to be understood [1]. For example the imitative behavior in newborn infants provides an innate foundation for understanding inner states belonging to other persons and for mapping others' actions into actions of their own body [2].

Since vision directly enables the contextual perception of surrounding objects, the absence of vision adversely affects not only language, motor, and cognitive development [3], but also social competence [4-6] in visually impaired people.

For example, since totally blind children lack visual references, they might not be aware of social cues in the form of body gestures while communicating with others. Similarly, low vision children might not correctly express and interpret body language because their imitative behaviors during infancy was prevented due to their degraded vision. As a consequence, visually impaired children often face problems in integrating socially. Preschool-age children with visual disabilities often have difficulty engaging in positive social interactions. Many do not display a full range of play behaviors [4-11], spend more time in solitary play interacting more with adults than with their sighted peers $[6,9,10,12$ 17], and are typically unable to demonstrate peer-related social competence [18-20]. This social impairment gives rise to feelings of frustration and fear of interaction, rather than selfefficacy and independence, which characterize the social experience of typical children.

Although coordinated and joint actions are critical to human social interaction [21, 22], no quantitative methods have been presented so far to evaluate social competences in visually impaired people. In the present study, we evaluated the level of social competence in visually impaired people through a coordinated action task, where the experimenter and the participant are one in front of the other while performing free hand movements. We applied the Granger causality analysis to the wrist movement kinematics and extracted the amount of 
information flow between the two agents [23, 24]. Information flow is a quantitative measure of how much one's movement influences some other's movement (see section II-E for a more detailed definition and to Supplementary Material for a mathematical definition).

Additionally, we evaluated whether visually impaired children improve their joint action coordination after a 12week interventional group program based on the Audio Bracelet for Blind Interaction (ABBI). ABBI is a technological bracelet and has been developed to enhance spatial and social cognition in visually impaired children through the strengthening of their audio motor associations [23].

\section{METHODS}

\section{A. Participants}

Six visually impaired ( 3 females, age range: mean age: 42 years old ) and eight sighted (4 females, age range: mean age: 40 years old) adults participated in the study. Furthermore, seventeen visually impaired (12 females, mean age: 12 years old) and twelve sighted (4 females, mean age: 13 years old) children aged 6 to 17 participated to the study. Adults and children groups were aged-matched $(t(12)=-0.15 ; \mathrm{P}=0.88$; $t(27)=0.35 ; \mathrm{P}=0.72)$.

The seventeen visually impaired children were recruited by the local rehabilitation centre for blind people (Istituto Chiossone, Genova) and also participated to the interventional program.

Every participant, or the parents of children participants, gave written consent before the experiments, as required by the Declaration of Helsinki and the local Ethics Committee (Comitato Etico ASL3 Genovese).

Visually impaired children and adults didn't have any additional sensory deficit besides blindness. The visual deficit was assessed according to the International Statistical Classification of Diseases and Related Health Problems (ICD) tenth revision [24]. In each case the visual deficit was of peripheral origin. The cognitive level of all the visually impaired children was assessed with the verbal scale of the 'Wechsler Intelligence Scale for Children - fourth edition (WISC-IV)' [25] and considered appropriate for their participation in the study, according to the cut off (>90) proposed by the authors for the composite score [26, 27]. Clinical details of the visually impaired participants are indicated in Table 1.

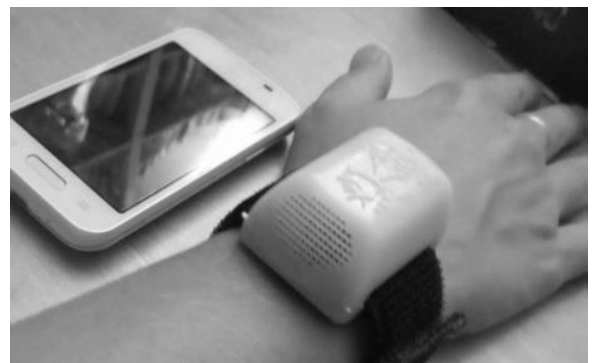

Figure 1. ABBI system. The system comprises the bracelet and a mobile phone with an ad-hoc application [28, 29].
TABLE I

CLINICAL DETAILS OF THE VISUALLY IMPAIRED PARTICIPANTS

\begin{tabular}{|c|c|c|c|c|}
\hline $\begin{array}{l}\text { Ge } \\
\text { nd } \\
\text { er }\end{array}$ & $\begin{array}{l}\text { Age } \\
\text { at } \\
\text { test }\end{array}$ & Pathology & $\begin{array}{l}\text { Age } \\
\text { complete } \\
\text { blindness }\end{array}$ & $\begin{array}{l}\text { Visual } \\
\text { acuity } \\
\text { (LogMAR) }\end{array}$ \\
\hline \multicolumn{5}{|c|}{ CHILDREN } \\
\hline M & 7 & Ocular albinism & Childhood & 1 \\
\hline M & 8 & Neurofibromatosis type I & Childhood & 1.7 \\
\hline M & 9 & Leber's amaurosis & Childhood & 1 \\
\hline $\mathrm{F}$ & 9 & Microphtalmia & Childhood & 1 \\
\hline $\mathrm{F}$ & 9 & Retinopathy of prematurity & Birth & $\begin{array}{l}\text { Light } \\
\text { perception }\end{array}$ \\
\hline $\mathrm{F}$ & 10 & Retinopathy of prematurity & Birth & None \\
\hline $\mathrm{F}$ & 10 & Retinopathy of prematurity & Birth & $\begin{array}{l}\text { Light } \\
\text { perception }\end{array}$ \\
\hline M & 11 & Tuberculous meningitis & Birth & $\begin{array}{l}\text { Light } \\
\text { perception }\end{array}$ \\
\hline $\mathrm{F}$ & 11 & Nystagmus & Childhood & 0.7 \\
\hline $\mathrm{F}$ & 12 & $\begin{array}{l}\text { Congenital cataract and } \\
\text { microphtalmia }\end{array}$ & Birth & $\begin{array}{l}\text { Light } \\
\text { perception }\end{array}$ \\
\hline $\mathrm{F}$ & 12 & Leber's amaurosis & Birth & $\begin{array}{l}\text { Light } \\
\text { perception }\end{array}$ \\
\hline M & 13 & Optic nerve glioma & Childhood & 0.7 \\
\hline $\mathrm{F}$ & 13 & $\begin{array}{l}\text { Stargardt's Macular } \\
\text { Distrophy }\end{array}$ & Childhood & 1 \\
\hline $\mathrm{F}$ & 14 & Optic nerve atrophy & Childhood & 1 \\
\hline $\mathrm{F}$ & 14 & Optic nerve atrophy & Childhood & 1.7 \\
\hline $\mathrm{F}$ & 15 & Retinopathy of prematurity & Birth & None \\
\hline $\mathrm{F}$ & 16 & Cones dystrophy & Birth & $\begin{array}{l}\text { Light } \\
\text { perception }\end{array}$ \\
\hline \multicolumn{5}{|c|}{ ADULTS } \\
\hline $\mathrm{F}$ & 27 & Retinopathy of prematurity & Birth & None \\
\hline $\mathrm{F}$ & 30 & Retinopathy of prematurity & Birth & None \\
\hline $\mathrm{F}$ & 33 & Congenital cataract & Birth & None \\
\hline M & 50 & Retinopathy of prematurity & Birth & None \\
\hline M & 57 & Optical uveitis & Birth & $\begin{array}{l}\text { Light } \\
\text { perception }\end{array}$ \\
\hline $\mathrm{M}$ & 58 & Congenital glaucoma & Birth & None \\
\hline
\end{tabular}

\section{B. $A B B I$}

$\mathrm{ABBI}$ is a wearable electronic device positioned on the wrist that produces sounds when moved. It (figure 1) comprises an ABBI bracelet and a mobile phone with an ad-hoc application that allows one to control the sound and the volume. The bracelet and the mobile phone communicate via Bluetooth Low-Energy [23, 28].

\section{Audio Social Interaction Task and Protocol}

The task was performed in a dark room for better tracking movements with the motion captive system. The task required the experimenter to wear ABBI on the wrist and move the arm making three different shapes (straight line, circle and lemniscate symbol) in random order. The participant stood in front of the experimenter and had to follow the experimenter movement that the participant perceived from the moving sound source (i.e. the ABBI bracelet). Each trial lasted 30 seconds, and each shape was performed 5 times in a random order, for a total of 15-20 minutes per participant including breaks. Blind and blindfolded sighted participants performed 15 trials in which the shape produced by the experimenter was known in advance and 6 trials in which it was not. Sighted participants also performed 15 trials without being blindfolded (figure 2) in order to have the baseline measurements for 
sighted healthy people and was always the last one, to avoid a learning effect due to vision.

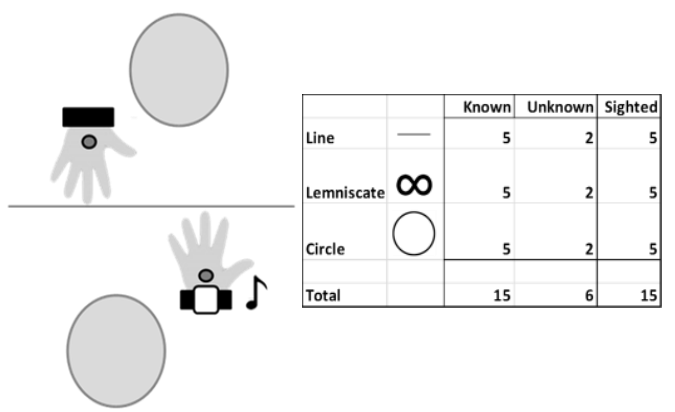

Figure 2. The audio social interaction task. Blind participants had a total of 21 trials, while sighted participant had a total of 36 trials.

A marker was positioned on the right wrist of both the experimenter and participant, and the movement kinematic was recorded using a motion capture system (Vicon, USA).

\section{Interventional program}

The visually impaired children also participated to a social interventional program. The program comprised three sessions: pre-evaluation, intervention, post-evaluation. The audio social assessment task was performed during the pre and post- evaluation sessions. The interventional session lasted 12 weeks, children met once per week in groups and performed a formal training with a professional therapist at the Istituto Chiossone for 45 minutes. Children were divided in 2 groups depending on their age. Group 1 included 9 children, age 7-11; while Group 2 included 8 children, age 12-16.

The ABBI device was used during the interventional session, which consisted in playing several social games that were adapted by the research group to visually impaired children. Depending on the game, children were required to wear the bracelet or localize the bracelet in the space interacting with all the other children (Table 2).

\section{E. Data Analysis and Granger Causality Analysis}

The ability of a participant to sustain interaction in the proposed tasks mostly depends on the information conveyed by $\mathrm{ABBI}$ from the experimenter to the participant. If such information is successfully transferred, then the experimenter's movements will affect the participant's movements (i.e., an information flow between the two will occur). Here we apply Granger Causality to quantitatively measure such information transfer (i.e., information flow). The registered cinematic data of the two wrists were reconstructed using the Vicon Nexus software (standard reconstruction) and exported in Matlab in the format of $[\mathrm{x}, \mathrm{y}, \mathrm{z}]$ matrices.

In order to measure the above described cause-effect relation we applied Granger causality (G-causality) analysis to the Matlab kinematic data [30]. G-causality is a statistical concept of causality based on prediction. Given a $x y$ time series pair, a time series $x$ is said to "Granger cause" a time series $y$, if the past values of $x$ provide statistically significant information to predict the next value of $y$. It follows that if a times series $x$ Granger causes a time series $y$ that implies that some
TABLE II

GROUP GAMES PERFORMED DURING THE REHABILITATION

\section{Home base or Home plate}

All children are assigned to two groups, each group having a leader who chooses the preferred sound of ABBI. A the same time both leaders turn on their ABBIs and the children belonging to their team have to localize the sound and reach the leader. The exercise ends when all children reach the correct position of the sound/leader.

Touch and find

All children are positioned in a circle. Each child in turn comes out from the circle and touch the shoulder of one of the other children. The two children walk following the perimeter of the circle and trying to find their own original places. To help them finding the original place, the two children positioned next to them turn on their ABBI providing them a sonorous cue.

Musical chairs

Several chairs are positioned in circle in the room (number of children minus one). All children wear ABBI and all ABBIs produce different sounds. The experimenter turns

the music on for several seconds, then he stops the music: when the music stops, all children have to find as soon as possible a free chair and sit down. At the end, one child will remain without a chair where to sit so he is excluded

from the following session. As the game goes on, one chair has to be removed from the circle.

Capture the flag

All children are assigned to two groups except one child who is the leader. Children in each group are assigned to a number and a specific sound. The leader has the flag and chooses a specific sound of ABBI, different from the other children participating. The leader calls a number and the children corresponding

to that number in each group has to localize the sound produced by the ABBI belonging to the leader, run towards the leader and capture the flag.

\section{Red light/ Green light}

All children line up on one side wearing ABBI, each child with a different sound. One child stays on the other side of the room and leads the game. When the leading child says 'green light', all the children run as fast as they can towards him, while when he says 'red light', everyone stops: whoever doesn't stop is out of the game. The leading child can recognize if a child did not stop in time thanks to the sound made by ABBI. The game continues until all the children are out or has gone from one end of the field back to where they started from.

Find your team-mate

All children are paired up: children belonging to a pair have ABBI set with

the same sound. The experimenter turns the music on until all children are separated from each other. When the experimenter stops the music, all children have to find their team-mate by localizing in the far space the same sound produced by their own ABBI.

Pass the object

The children are seated in a circle and they have to pass a sounding ABBI to their closest pair in a clockwise order.

Scavenger hunt

Several ABBIs are hidden in the room. When the game starts, each ABBI in turn is switched on and the children have to localize it.

Swap the chair

All children seat in a circle, but two children at a time play the game. The experimenter calls two children: these children have to stand up, take off their ABBIs and leave them on the chair. The experimenter then activates the ABBIs and each child has to find the chair belonging to the other child by following the sound produced by ABBI.

Lightningbusters

The children are assigned to one of three different roles: the lightningbuster (who makes the other children immobile when he touches them), the saviours (who make the paralyzed children free when they touch him), and the fugitives (who run away from the lightningbuster until he finds them). All the children except the lightningbuster don't wear ABBI and they all run in the room. As soon as the lightningbuster touches a child, the child immediately freezes. All the saviors can try to find the frozen child and make him free by touching him again. 
information has been transferred from $x$ to $y$ affecting the behavior of $y$.

In its standard and linear formulation, G-causality is based on linear Auto-regressive (AR) models for prediction, for an overall 4 AR models for each $x y$ time series pair. For details, and for a brief discussion on similarities between G-causality and Information Theory measures, please refer to Supplementary Material.

As for time series for G-causality analysis, we used the instantaneous velocity magnitude of the wrist movements. The velocity magnitude provides a single informative measure to represent one person wrist movement, as opposed to, e.g., 3D trajectories that would require much more complex analyses (e.g., to handle multi-variate time series). Additionally, although the velocity magnitude does not provide the precise information about the "depicted" figure (e.g., line, circle or lemniscate) that 3D trajectories provide, each figure has its own characteristic velocity profile.

Missing values were interpolated through a polynomial cubic interpolation.

To handle the overall non-stationarity of the velocity within a trial, each time series was segmented into 5 -second windows shifted every second. Then, AR models and G-causality were computed at each window.

Our measure to assess the interaction between participant and experimenter is referred to as (per-trial) information flow which is defined as the ratio between the no. of windows in which the experimenter time series (i.e., velocity) significantly G-causes the participant time series, and the overall number of windows (see Supplementary Material). We additionally computed the overall information flow defined as experimenter-to-participant flow plus participant-toexperimenter flow.

G-causality was computed using the off-the-shelf GCA Matlab toolbox [31].

\section{F. Statistics}

Data are presented as mean and standard deviation (SD). To evaluate the performance of the adult groups, two 2-ways mixed effect ANOVAs (one for each indicator) were performed with factors Group (Blind, Sighted, Blindfolded), and Shape (known, unknown). Two further ANOVAs with factor Group (Blind, Sighted) was performed to compare the two groups. To evaluate the performance of the children groups, two 2-ways mixed effect ANOVAs (one for each indicator) were performed with factors Group (Blind, Sighted, Blindfolded), and Shape (known, unknown). Two further ANOVAs with factor Group (Blind, Sighted) was performed to compare the two groups. To evaluate the effect of the group intervention in blind children, data were subjected to separate two-tailed paired samples t-tests. The threshold for statistical significance was set to $P<0.05$.

\section{RESULTS}

Results are presented in figure 3 .

\section{A. Visually impaired and sighted blindfolded adults}

The mixed effect ANOVA, the factor Group resulted significant $\left(\mathrm{F}_{(1,38)}<58.01, \mathrm{P}<0.001\right)$, for both the Experimenter to Participant and Overall coefficient. Looking at the Experimenter to Participant coefficient, the mean value for Blind adults was 0.019 , while for Blindfolded was doubled at 0.039 and for Sighted was almost tripled at 0.052. No significant difference was present between the knowing and not knowing the shape in advance. However, in the condition of shape unknown in advance, the ratio in both indicators showed a trend to be lower.

\section{B. Visually impaired and sighted blindfolded children}

Both the factorial ANOVAs resulted significant for the main effect Group $\left(\mathrm{F}_{(1,38)}<57.01, \mathrm{P}=0.001\right)$.

Visually impaired children have lower capability to perform coordinated actions with another peer (in this case the experimenter) when compared to sighted children. In fact, regarding the Experimenter to Participant coefficient the ratio of Granger Casuality children is lower in both the case of shape known or unknown in advance. The mean value of sighted children is doubled in the blindfolded condition (0.019 vs 0.038$)$ and even bigger in the sighted condition (0.053). The overall coefficient results even 4 times bigger for the sighted condition in comparison to blind, peaking at 0.082 . Interestingly, regarding the Overall coefficient, it seems that the overall flow is also influenced by the ability of the Experimenter to follow the Participant, even if no statistical difference was present. This results is quite intuitive as during the task, the Experimenter was leading the movement.

\section{Social interventional program with $A B B I$}

By comparing the outcomes of the auditory tasks described above before and after the dedicated training with ABBI, we found a general improvement of the auditory spatial performance in all children enrolled in the interventional program in both the case of shape known in advance ( $t=-$ 4.063, $\mathrm{P}<0.0002)$ or shape unknown in advance $(\mathrm{t}=-5.667$, $\mathrm{P}<0.0001)$. In fact, after the interventional session, the range of ratio of Granger casuality in visually impaired children resulted similar to the one of sighted children not blindfolded. 
A
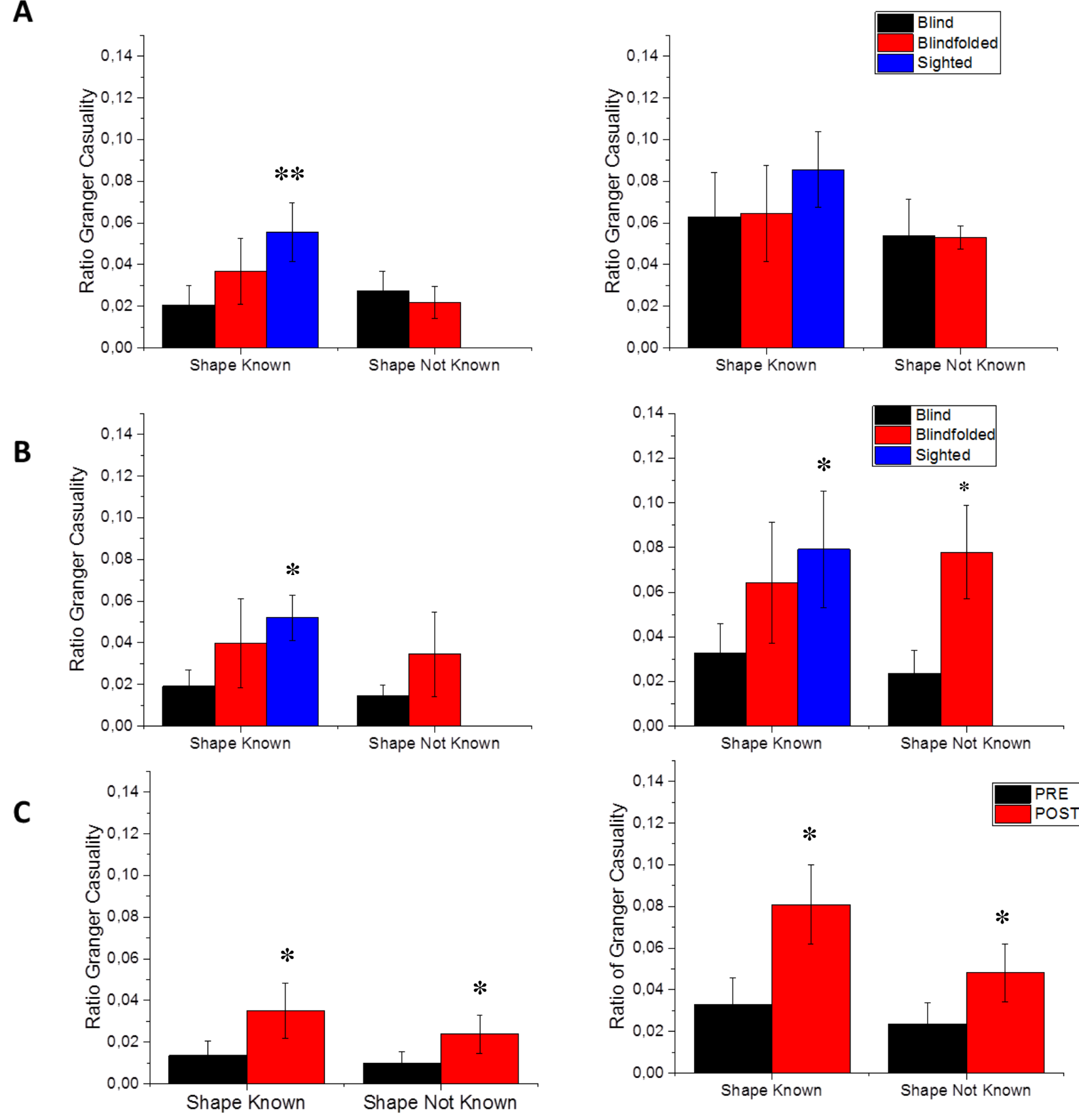

Experimenter to Participant

Overall

Figure 3: The ratio of granger causality is shown to represent the flow of information from the experimenter to the participant (left panel) and the overall flow of information between the two agents (right panel). A. Ratio of Granger causality in visually impaired and sighted adults. B. Ratio of Granger causality in visually impaired and sighted children. C. Ratio of Granger causality relative to the social interventional program for 7 to 17 years old visually impaired children. Significant differences are indicated as $* \mathrm{P}<0.01$ and $* * \mathrm{P}<0.001$. 


\section{CONCLUSION}

The acquisition of spatial hearing is typically a good indicator of the future ability to independently navigate in the environment and engage in positive social interaction with peers. While for sighted individuals the visual feedback represents the most important incentive for actions and thus for the development of mobility and social skills [32], visually impaired individuals strongly rely on auditory landmarks to encode spatial and social information. Indeed, it has been shown that visually impaired children often have difficulties engaging in positive social interactions, making their assimilation into preschool programs difficult and giving rise to counter-productive feelings of frustration.

In this study, we proposed an audio social interaction task in which two agents have to interact thanks to an audio source in order to synchronize their arm movements. As a measure of social competence, the application of the Granger Causality method allows the quantification of sensorimotor information flow between partners. This is the first attempt at the evaluation of joint action coordination in visually impaired people. More importantly, we also measured the effects of an extensive but entertaining interventional program with ABBI on the development of social competence in visually impaired children aged 7-17 years old.

Our results indicate that the flow of communication between the two agents is lower in visually impaired people, especially considering the flow transferred from the experimenter to the visually impaired (experimenter to participant coefficient).

Furthermore, the flow of information from the experimenter to the child is increased after the social training with ABBI both for the case in which children knew in advance the shape that the experimenter was performing or in the case were the shape was unknown.

A key limitation of the present interventional program is the lacking of a control group. However, the impressive increase in information flow suggests the capability to decode audio movements and to interact with it can be improved with entertaining activities carried out with new technological devices as ABBI. The test proposed here can be a trading point for the early assessment of social abilities in visually impaired children. This will allow to develop adequate intervention programs to restore or rehabilitate impaired aspects of social perception in visually impaired people.

\section{ACKNOWLEDGMENT}

Authors thank all the children and they family that participated in the experiment. We thank all the children participated at this study and the Chiossone rehabilitators Marika Rolando, Debora Grammatico, and the MD Elisabetta Capris for the important contribution, discussion and work performed during the interventional program with children. We thank the EDL (Electronic Design Facility of IIT) facility for the work done for the ABBI device. We thank all the
ABBI consortium for the work done associated with the development of the ABBI device and applications.

\section{REFERENCES}

[1] M. Groenveld, "Effects of visual disability on behaviour and the family," Clinics in developmental medicine, pp. 64-77, 1993.

[2] A. N. Meltzoff and J. Decety, "What imitation tells us about social cognition: a rapprochement between developmental psychology and cognitive neuroscience," Philosophical Transactions of the Royal Society of London B: Biological Sciences, vol. 358, pp. 491500, 2003.

[3] C. Gilbert and H. Awan, "Blindness in children-half of it is avoidable, and suitable cost effective interventions are available," Bmj, vol. 327, pp. 760-761, 2003.

[4] M. J. Guralnick, Assessment of peer relations: Center on Human Development and Disability, University of Washington, 1992.

[5] M. J. Guralnick, R. T. Connor, M. Hammond, J. M. Gottman, and K. Kinnish, "Immediate effects of mainstreamed settings on the social interactions and social integration of preschool children," AJMR-American Journal on Mental Retardation, vol. 100, pp. 359-377, 1996.

[6] M. Rettig, "The play of young children with visual impairments: Characteristics and interventions," Journal of Visual Impairment \& Blindness, 1994.

[7] M. J. Guralnick, R. T. Connor, M. A. Hammond, J. M. Gottman, and K. Kinnish, "The peer relations of preschool children with communication disorders," Child Development, vol. 67, pp. 471489, 1996.

[8] M. J. Guralnick, J. M. Gottman, and M. A. Hammond, "Effects of social setting on the friendship formation of young children differing in developmental status," Journal of Applied Developmental Psychology, vol. 17, pp. 625-651, 1996.

[9] S. Sacks and L. Kekelis, The development of social skills by blind and visually impaired students: Exploratory studies and strategies: Amer Foundation for the Blind, 1992.

[10] S. Sacks, L. Kekelis, and R. Gaylord-Ross, "The Development of Social Skills," New York, NY: American Foundation for the Blind, 1992.

[11] S. R. McConnell and S. L. Odom, "A multimeasure performancebased assessment of social competence in young children with disabilities," Topics in Early Childhood Special Education, vol. 19, pp. 67-74, 1999.

[12] E. Adelson and S. Fraiberg, "Gross motor development in infants blind from birth," Child development, pp. 114-126, 1974.

[13] E. Anderson and L. Kekelis, "Language input and language acquisition: Evidence from special populations," in 17th annual Child Language Research Forum, Stanford University, Stanford, CA, 1985.

[14] S. Parsons, "Function of play in low vision children: II. Emerging patterns of behavior," Journal of Visual Impairment \& Blindness, 1986.

[15] A. C. Skellenger and E. W. Hill, "Effects of a shared teacher-child play intervention on the play skills of three young children who are blind," Journal of Visual Impairment \& Blindness, 1994.

[16] H. Troster, W. Hecker, and M. Brambring, "Longitudinal study of gross-motor development in blind infants and preschoolers 1," Early Child Development and Care, vol. 104, pp. 61-78, 1994.

[17] D. H. Warren, "Blindness and early childhood development," 1977.

[18] M. Celeste, "Social skills intervention for a child who is blind," Journal of Visual Impairment \& Blindness, vol. 101, p. 521, 2007.

[19] E. P. Simon and A. E. Gillman, "Mainstreaming visually handicapped preschoolers," Exceptional children, 1979.

[20] P. E. Tait and C. Wolfgang, "Mainstreaming a blind childProblems perceived in a preschool day care program," Early Child Development and Care, vol. 13, pp. 155-167, 1984. 
M. Tomasello, M. Carpenter, J. Call, T. Behne, and H. Moll, "Understanding and sharing intentions: The origins of cultural cognition," Behavioral and brain sciences, vol. 28, pp. 675-691, 2005.

[22] C. A. Brownell, "Early developments in joint action," Review of philosophy and psychology, vol. 2, pp. 193-211, 2011.

[23] S. Finocchietti, G. Cappagli, G. Baud-Bovy, C. Magnusson, H. Caltenco, G. Wilson, et al., "ABBI, a New technology for sensorymotor rehabilitation of visual impaired people," in International Conference on Enabling Access for Persons with Visual Impairment, 2015, pp. 80-84.

[24] W. H. Organization, International statistical classification of diseases and related health problems vol. 1: World Health Organization, 2004.

[25] D. Wechsler, "Wechsler intelligence scale for children," 1949

[26] L. G. Weiss, D. H. Saklofske, A. Prifitera, and J. A. Holdnack, WISC-IV advanced clinical interpretation: Academic Press, 2006.

[27] J. M. Sattler, Assessment of children: Cognitive foundations: JM Sattler San Diego, CA, 2008.

[28] B. L. Porquis, S. Finocchietti, G. Zini, G. Cappagli, M. Gori, and G. Baud-Bovy, "ABBI: A Wearable Device for Improving Spatial and Social Cognition in Visually-Impaired Children," presented at the IEEE Biomedical Circuits and Systems Conference (BIOCAS), Turin, 2017.

[29] S. Finocchietti, G. Cappagli, L. Porquis, G. Baud-Bovy, E. Cocchi, and M. Gori, "Evaluation of the Audio Bracelet for Blind Interaction for improving mobility and spatial cognition in early blind children - A pilot study," in Engineering in Medicine and Biology Society (EMBC), 2015 Annual International Conference of the IEEE, 2015, pp. 7998-8001.

[30] C. Granger, "Investigating causal relations by econometric models and cross-spectral methods. ," Econometrica pp. 424-438, 1969.

[31] A. K. Seth, "A MATLAB toolbox for Granger causal connectivity analysis. ." J Neurosci Meth pp. 262-273, 2010.

[32] M. Gori, G. Cappagli, A. Tonelli, G. Baud-Bovy, and S. Finocchietti, "Devices for visually impaired people: high technological devices with low user acceptance and no adaptability for children.," Neurosci. Biobehav. Rev, vol. 69 pp. 79-88

2016.

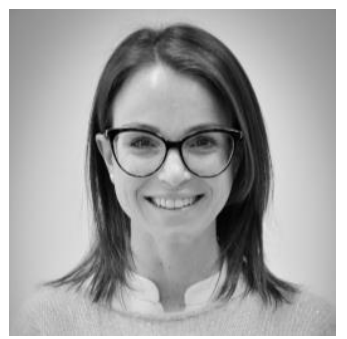

Giulia Cappagli received her Msc. in Psychology from the University of Florence (2012) and Ph.D. in Bioengineering and Robotics from the Italian Institute of Technology (2017) on assessing and rehabilitating spatial perception in children and adults with visual impairments. During her studies she has been a visiting researcher at the University College London (2012), and University of Oxford (2016). Giulia is currently working on Horizon2020 weDRAW project which aims at exploiting the best sensory modality for learning arithmetic and geometrical concepts based on multisensory interactive ICTs and serious games.

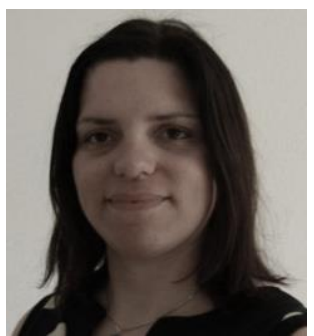

Sara Finocchietti is a Senior Postdoc within the Unit for Visually Impaired People at the Italian Institute of Technology (IIT) since 2014. She received her Msc. in Biomedical engineering from the University of Genoa, Italy (2007) and $\mathrm{Ph} . \mathrm{D}$. in Clinical Science from Aalborg University, Denmark (2011). She has been also guest researcher in Nagoya University (2010) and Goettingen University (2012) working on sensory assessment. Her research interests cover a wide range of topics as technology development, sensory assessment, rehabilitation, and cognitive neuroscience.

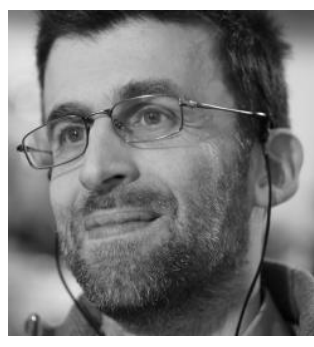

Gabriel Baud-Bovy is Assistant Professor at the Faculty of Psychology of the San Raffaele University since 2003 and Researcher at the Italian Institute of Technology in Genova since 2011. His reseach interests are centered on haptic perception and exploration of the environment, the control of finger forces in multi-finger grasp, human-human and human machine physical interaction and, more generally, the development and application of haptic technology and methods.

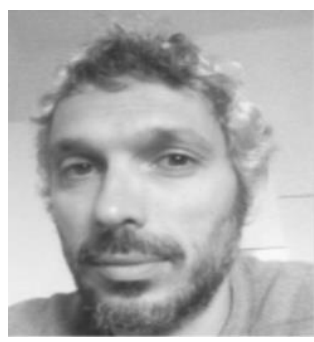

Leonardo Badino is a Researcher at the Center for Translational Neurophysiology of Speech and Communication at the Italian Institute of Technology (IIT). He received a 5-year degree (BEng + MEng) in Electronic Engineering from the University of Genova (1994-2000) and a $\mathrm{PhD}$ in Computer Science from University of Edinburgh (2006-2010). Before moving to Edinburgh he worked as software engineer/project manager at Loquendo, a speech technology company (2001-2006). His main research interests include automatic speech recognition (ASR) with a focus on neuro-inspired ASR, machine learning for speech and language processing, text-to-speech synthesis, and analysis of sensorimotor communication.

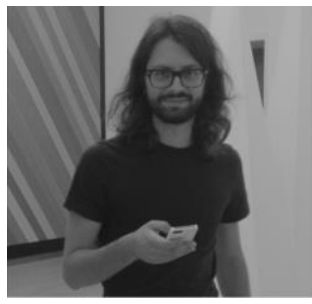

Alessandro D'Ausilio is Associate Professor of Physiology (since 2017) at the University of Ferrara and Researcher at the Italian Institute of Technology (since 2012). He holds an Experimental Psychology degree (2003) and a Ph.D. in Cognitive Psychology from the Sapienza University of Rome (2007). During the $\mathrm{PhD}$ he spent one year at the EberhartKarls-Universität in Tübingen (2004) and one at the MIT (2005). His first Post-Doc was at the University of Ferrara (2007-2010), while the second was at IIT in Genova (2010-2012). In 2016 he moved to the IIT Center of Translational Neurophysiology for Speech and Communication, hosted by the University of Ferrara. His research interests cover the neurophysiology of motor control, the role of motor processes in action and speech perception as well as the investigation of verbal and non verbal joint action.

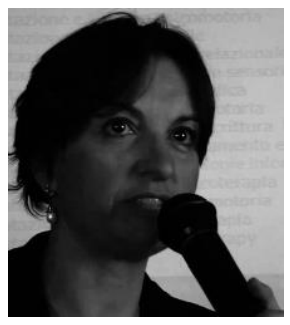

Elena Cocchi (M.D.) obtained her degree in Medicine and Surgery in 1997 and her degree in Clinical Psychology in 2002. Between 2003 to 2007 she was Research fellow at the Child Neuropsychiatry Unit of Istituto G.Gaslini, Genoa, Italy. Since May 2009 she is Director of the Childhood Unit at the Istituto David Chiossone per ciechi ed ipovedenti, Genoa.

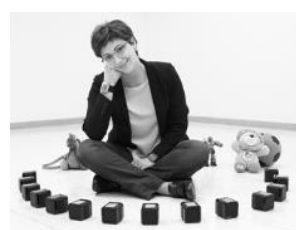

Monica Gori obtained received her Msc. in psychology in 2004 from the University of Florence. She then spent 3 years at the CNR in Pisa in the Neuroscience group guided by prof. David Burr. She obtained her Ph.D. in human technologies in 2009 from the Italian Institute of Technology under the supervision of Prof. Giulio Sandini. After a period in Berkeley (USA), she is now tenure-track and principal investigator of the Unit for Visually Impaired People at IIT. Her research group aims at studying the development of children with and without disability and developing innovative rehabilitative technologies. She coordinated the FP7 project ABBI and is now coordinating the Horizon 2020 project weDraw. 